Review Article

\title{
Biosynthesis of Structurally Diverse Triterpenes in Plants: the Role of Oxidosqualene Cyclases
}

\author{
SUMIT GHOSH* \\ Biotechnology Division, Council of Scientific and Industrial Research-Central Institute of Medicinal \\ and Aromatic Plants, Lucknow 226 015, India
}

(Received on 23 November 2015; Revised on 04 March 2016; Accepted on 16 March 2016)

\begin{abstract}
Triterpenes, thirty-carbon compounds derived from five-carbon isoprene units, are among the largest and diverse classes of natural products. To date, over 20,000 distinct triterpene structures having more than 100 skeletal variations are indentified from natural sources. The majority of this triterpene diversity is found within the plant kingdom. Plants often accumulate triterpenes in their glycosylated forms that are known as saponins. Plants usually produce triterpenes in tissue-specific, organ-specific and developmentally specific manners, and in response to environmental perturbation, and pest and pathogen attacks. Although, the biological function of majority of the triterpenes is yet to be determined, some triterpenes are suggested to play specialized/secondary function as defence compounds. On the contrary, sterols and steroid hormones that share common biosynthetic precursor (i.e. 2,3-oxidosqualene) with triterpenes play primary function during plant growth and development. The first diversifying step in triterpene/sterol biosynthesis is catalyzed by a family of enzymes known as oxidosqualene cyclases (OSCs) that convert 2,3-oxidosqualene into various triterpene/sterol scaffolds. In recent years, remarkable progress has been made in enzymatic characterization of OSCs from diverse plant species, besides isolation, structural elucidation and studying pharmacological activities of triterpenes. These efforts led to the identification of OSCs with diverse product specificities and, also helped us understand the evolution of OSC family in plants. In this review article, besides presenting an overview of triterpenes with respect to their structural diversity, biological functions and biosynthetic pathways, the role of OSCs in generating triterpene skeletal diversity in plants is highlighted.
\end{abstract}

Keywords: Natural Product; Phytochemical; Metabolite; Terpene; Triterpene; Sterol; Oxidosqualene Cyclase

\section{Introduction}

Plants biosynthesize and accumulate a diverse type of low-molecular-weight organic compounds. Majority of these natural products/phytochemicals are biosynthesized and accumulated in plants in a subcellular compartment/tissue/developmental age/ species-specific manner (Misra et al., 2015). More than 100,000 different structures of natural products are revealed. However, the actual number is certainly more because only about $30 \%$ of the plant species are studied for the purpose of natural products isolation and characterization (Wink, 2010). Unlike primary metabolites; such as amino acids, sugars, lipids and nucleic acids, the majority of the phytochemicals do not play direct role in plant growth and development. However, they help plants to acclimatize to the demanding environmental conditions and therefore, referred as secondary or specialized metabolites (Gershenzon and Dudareva, 2007). The biological functions of plant secondary metabolites include attracting pollinators/seed dispersal agents, interacting with neighbouring plants, and responding to the attacks by pathogens/herbivores (Pichersky and Gershenzon, 2002).

A vast diversity of the plant secondary metabolites has been exploited for thousands of years by the human cultures to treat several diseases and health disorders, and also used as flavours, fragrances and pesticides. Majority of the world's population is still dependent on plants or plant extracts as a prime source of healthcare. More than half of the top 150 prescribed drugs in the US comprise of at least one

*Author for Correspondence: E-mail: sumitghosh@cimap.res.in; sumitg80@gmail.com 
plant-derived compound. In India, traditional medicine is still practiced by up to $80 \%$ of the population (Williams and Ellis, 2013). At present, several plantorigin secondary metabolites are directly utilized as drugs; however, many are leading models for the development of semi-synthetic and synthetic drugs (Canter et al., 2005; De Luca et al., 2012). Some of the plant-origin life-saving drugs are in extensive medical use for the treatment of malaria (artemisinin, quinine), cancer (vinblastine, vincristine, taxol), hypertension (reserpine), pain (morphine, codeine) etc. Moreover, several phytochemicals including farnesane, bisabolene, pinene dimmers, isopentenal and botryococcene are emerging as valuable biofuel feedstocks (Jiang et al., 2016).

According to the biosynthetic origin, natural products are grouped into three major classes terpenes (derived from five-carbon isoprene units), alkaloids (nitrogen-containing compounds derived from amino acids) and phenylpropanoids (derived predominantly from phenylalanine and, to a lesser extent from tyrosine). Among these three classes, terpenes constitute the largest family of natural products with more than 60,000 members (Koksal et al., 2011). Terpenes are further classified into different subgroups, based on the number of five-carbon (5C) isoprene units they contain. Hemiterpenes contain a single isoprene unit, whereas; mono (10C)-, sesqui (15C)-, di (20C)-, sester (25C)-, tri (30C)-, tetra (40C)and poly-terpenes are made of two, three, four, five, six, eight and more than eight isoprene units, respectively. Majority of low-molecular-weight terpenes such as hemiterpenes (e.g. isoprene), monoterpenes (e.g. geraniol), and sesquiterpenes (e.g. farnesene) are volatile in nature and are emitted from plants to attract/repel other organisms during interaction with the environment. However, highmolecular-weight terpenes, such as diterpenes (e.g. taxol), triterpenes (e.g. ursolic acid), tetraterpenes (e.g. carotenoid) and polyterpenes (e.g. rubber) are non-volatile and therefore, most of them are believed to play localized biological function.

Triterpenes are one of the diverse groups of natural products with more than 20,000 different triterpene structures reported to date (Thimmappa et al., 2014). Triterpene class of secondary metabolites have commercial applications for their diverse pharmacological activities and, also as food and cosmetic additives (Laszczyk, 2009; Sawai and Saito, 2011; Moses et al., 2013). Several plant-origin triterpenes are now being marketed as dietary supplements and over-the-counter (OTC) drugs, and a couple of synthetic triterpene derivatives are undergoing clinical trials (Sheng and Sun, 2011; Moses et al., 2013). Unfortunately, secondary metabolites including triterpenes are produced at a very low level as a mixture of structural/stereo-isomers in plants. Therefore, complex extraction and isolation procedures are followed, which lead to low product yield. A comprehensive knowledge about the biosynthetic pathways and their regulation shall be useful to overcome low product yield of the secondary metabolites and also to design a specific chemotype, following pathway engineering and molecular breeding approaches (Lange and Ahkami 2013; Paddon et al., 2013). 2,3-Oxidosqualene serves as a common precursor for the biosynthesis of diverse triterpenes in plants. Oxidosqualene cyclase (OSC)-mediated cyclization of 2,3-oxidosqualene is the initial diversifying step for the biosynthesis of diverse triterpenes (Misra et al., 2014). Further, cytochrome P450-dependent monooxygenase (CYP450), acyltransferase (AT) and glycosyltransferase (GT)mediated oxidation, acetylation and glycosylation, respectively, of the product of OSC-catalyzed reaction lead to generation of additional triterpene structural diversity. This review article presents an overview of triterpenes with respect to the structural diversity, biological roles and biosynthetic pathways. Moreover, an important role of OSCs in generating triterpene diversity is highlighted.

\section{Triterpene Structural Diversity}

Triterpenes constitute large and structurally diverse types of natural products. More than 100 distinct triterpene scaffolds are currently known. These scaffolds are found to be decorated with various functional groups; such as hydroxyl, carboxyl, carbonyl, alkyl, malonyl and glycosyl to produce over 20,000 distinct triterpene structures that are known to date (Thimmappa et al., 2014). Triterpenes are derived from either of two acyclic 30-carbon precursors: squalene (bacteria) and 2,3-oxidosqualene (fungi, plants and animals). These are also common precursors for the biosynthesis of membrane sterols, such as bacterial hopanoids, plant phytosterols, animal cholesterol and fungal ergosterol, and steroid 
hormones such as plant brassinosteroids, animal corticosteroids and insect ecdysone (Bishop and Koncz, 2002). Most triterpene scaffolds are tetracyclic and pentacyclic in nature, however, acyclic, monocyclic, bicyclic, tricyclic, and hexacyclic triterpenes have also been reported from natural sources. Based on their carbon skeleton, triterpenes are commonly classified as squalene derivative, cucurbitane, cycloartane, dammarane, euphane, friedelane, gammacerane, holostane, hopane, lanostane, lupane, oleanane, protostane, quassinoid, saponin, serratane, tetranortriterpene, tirucallane and ursane (Hill and Connolly, 2012). These triterpene classes (Fig. 1) are briefly discussed in the following sections.

\section{Acyclic Triterpene}

Squalene and squalene epoxide (2,3-oxidosqualene) are acyclic triterpenes that serve as general precursors for the biosynthesis of diverse triterpenes in bacteria and eukaryotic organisms, respectively. However, plants also produce acyclic triterpenes which are known as squalene derivatives (Fig. 1). Examples are sapelenins isolated from bark of the Cameroonian medicinal plant Entandrophragma cylindricum and callicarpol isolated from dried fruit of Callicarpa macrophylla (Hill and Connolly, 2012). Some of the acyclic triterpenes such as ekeberins isolated from stem bark of Ekebergia capensis, an African medicinal plant, are the potential anti-malarial compounds (Amoa Onguéné et al., 2013). Another squalene derivative, squalene-1,10,24,25,30-pentol that exhibits moderate anti-mycobacterial activity, has been isolated from leaves and twigs of Rhus taitensis (Hill and Connolly, 2013). Cupaniol isolated from Cupania latifolia and lobophytene isolated from the Vietnamese soft coral Lobophytum sp. are also classified as squalene derivatives. Other acyclic triterpenes are botryococcene and their methylated derivatives produced by the green algae Botryococcus braunii (Jiang et al., 2016).

\section{Monocyclic, Bicyclic and Tricyclic Triterpene}

Triterpenes having one-to-three ring structure are also found in plants, although less abundant compared to triterpenes with four-to-five ring structure (Fig. 1). Monocyclic triterpenes achilleol A and camelliol C are isolated from Asteridae subclass members Achillea odorata and Camellia sasanqua (Xu et al., 2004). Other monocyclic triterpenes are iristectorone $\mathrm{K}$ (a triterpene ester) and monocyclosqualene that were isolated from rhizomes of Iris germanica (Orhan et al., 2002) and leaves of Ligularia fischeri (Lee et al., 2002), respectively. Myrrhanones, (+)-myrrhanol C, lamesticumins, lansic acid and its 3-ethyl ester are the examples of bicyclic triterpenes reported from Commiphora mukul, Pistacia lentiscus and Lansium domesticum (Dong et al., 2011; Mallavadhani et al., 2015). Among these bicyclic triterpenes, myrrhanone $\mathrm{C}$ derivatives and (+)myrrhanol $\mathrm{C}$ are the potential anti-cancer leads (Domingo et al., 2013; Mallavadhani et al., 2015). Tricyclic triterpenes are also reported from plants, such as Kadsura coccinea, A. odorata and Schisandra glaucescens containing kadcotriones, achilleol B and schiglautone A, respectively (Barrero et al., 1990; Meng et al., 2011; Liang et al., 2013).

\section{Tetracyclic Triterpene}

Tetracyclic triterpenes are frequently classified as lanostane, protostane, dammarane, euphane and tirucallane (Fig. 1). Examples of lanostane-type tetracyclic triterpenes are lanosterol and parkeol. Lanosterol is the universal precursor for the biosynthesis of sterols in animals (cholesterol) and fungi (ergosterol), however, lanosterol-derived metabolites are also found in plants and some myxobacteria (Bode et al., 2003; Kolesnikova et al., 2006). Parkeol serves as sterol precursor in sea cucumbers and is a wide-spread metabolite in plants (Xu et al., 2004). Cucurbitane, euphane, tirucallane and holostane are lanostane derived triterpenes. Cucurbitacins are widespread in cucurbits such as cucumber, melon, watermelon, squash, and pumpkin, and are considered as responsible for the bitter taste of these plants. Cucurbitacins are suggested to protect plants from most pests and also shown to have antitumor properties (Shang et al., 2014). Euphane and tirucallane triterpenes are reported from roots of Euphorbia kansui and from bark of Broussonetia papyrifera (Wang et al., 2003; Hill and Connolly, 2013). Holostanes, such as cucumariosides and fuscocinerosides are commonly found in sea cucumber (Hill and Connolly, 2013). Dammaranes are the well known bioactive components of the medicinal plants Panax ginseng and $P$. notoginseng. These plants accumulate dammarane glycosides, such as ginsenosides, notoginsenosides and gypenosides. 


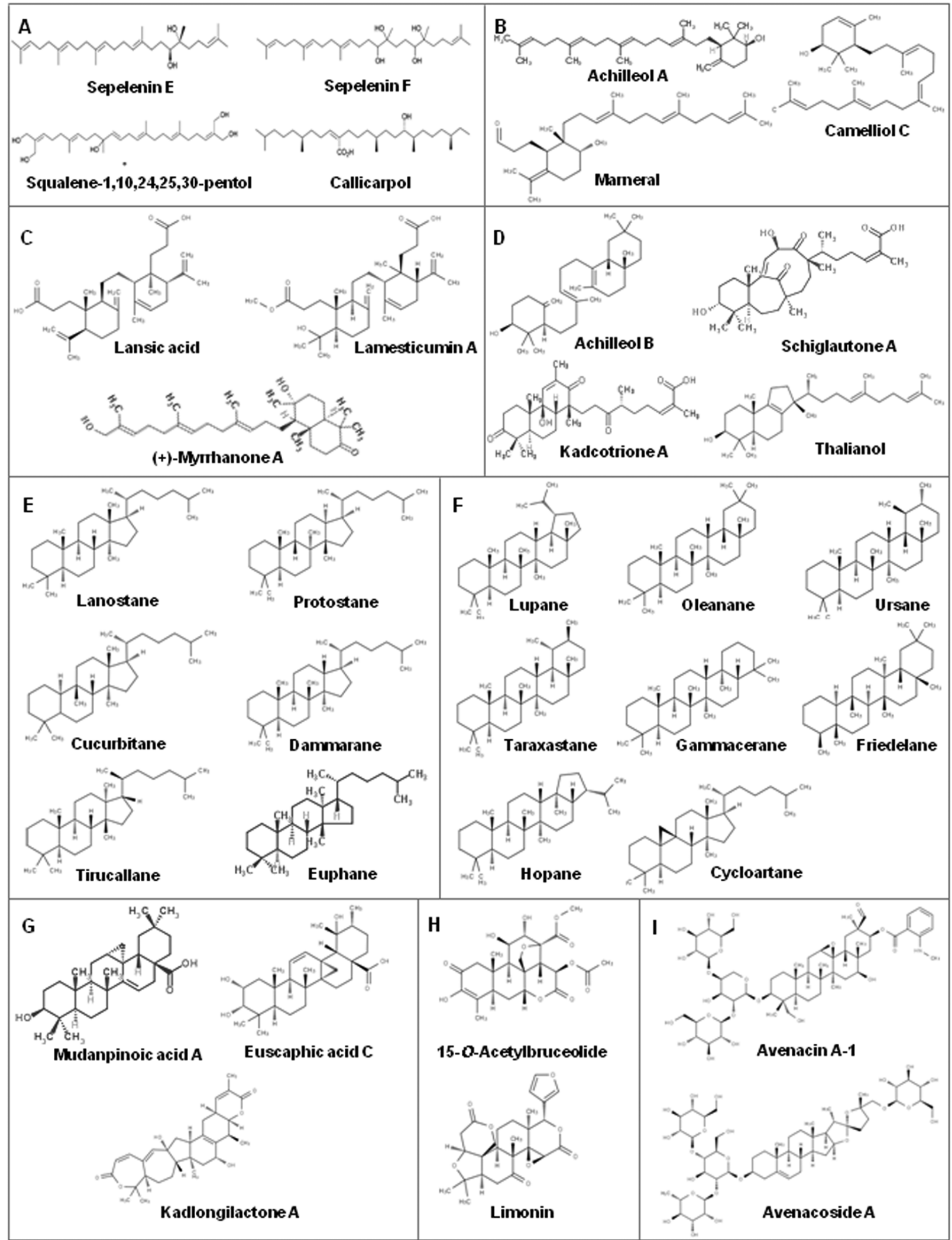

Fig. 1: Triterpene skeletal diversity. (A-G) Acyclic-to-hexacyclic triterpenes, (H) quassinoid (15-O-acetylbruceolide) and tetranortriterpene (limonin), (I) saponins. Structures are obtained from Chemical Entities of Biological Interest (ChEBI, http://www.ebi.ac.uk/chebi), ChemSpider (http://www.chemspider.com) and cited references 
Dammarane glycosides are also reported to present in other plant species: centellosides from Centella asiatica and betalnosides from Betula alnoides (Hill and Connolly, 2013). Protostane is a stereoisomer of dammarane. More than fifty protostane structures are known in nature. Protostanes are primarily limited to the genus Alisma of the Alismataceae family of aquatic plants, although, these triterpenes are also infrequently found in plants of the other genera such as Lobelia, Garcinia, and Leucas. Some of the protostanes, such as alisol A derivatives are recognised for their anti-microbial and anti-cancer activities (Zhao et al., 2013).

\section{Pentacyclic Triterpene}

Pentacyclic triterpenes are known for the variety of pharmaceutical activities, such as anti-inflammatory, hepatoprotective, anti-cancer, anti-hyperlipidemic, anti-diabetic, anti-oxidant and anti-microbial (Laszczyk, 2009; Kong et al., 2013; da Silva Ferreira et al., 2013). Pentacyclic triterpenes are now being marketed as dietary supplements, as well as, overthe-counter (OTC) drugs, and some of the synthetic derivatives are undergoing clinical trials (Sheng and Sun, 2011; Moses et al., 2013). Pentacyclic triterpenes are commonly classified as friedelane, gammacerane, hopane, lupane, oleanane, serratane, taraxastane and ursane (Fig. 1). Lupane, oleanane and ursane are the three major classes of pentacyclic triterpenes widely distributed in plants. The examples of the plant-origin lupane-type pentacyclic triterpenes are betulinic acid, bacosine, bevirimat, melaleucic acid, cylicodiscic acid, calenduladiol, glochidonol etc. Among these, betulinic acid, bevirimat and bacosine are well known for their pharmacological activities, including; anti-cancer, anti-HIV and anti-hyperglycemic activities (Martin et al., 2008; Ghosh et al., 2011; Potze et al., 2014). Bacosine and bevirimat have a limited species distribution and predominantly found in Bacopa monnieri and Syzygium claviflorum, respectively. However, betulinic acid is widely distributed in plant species including Betula sp. after which this compound was named.

Oleanolic acid, maslinic acid, arjunic acid, glycyrrhetinic acid, germanicol, moronic acid, quillaic acid, and hederagenin are the examples of oleananetype pentacyclic triterpenes. Oleananes are generally widely distributed among plant species, however, few oleananes have limited species distribution, such as; arjunic acid in Terminalia arjuna and glycyrrhetinic acid in Glycyrrhiza sp. Oleanolic acid is one of the most widely distributed triterpenes in plants. Oleanolic acid has been isolated from more than 1600 plant species. However, it is more prevalent in the plant species of Oleaceae family, including Olea europaea, based on which it was named (Pollier and Goossens, 2012). Oleanolic acid is also widely distributed among the Lamiaceae family members including Ocimum species (Misra et al., 2014). Oleanolic acid and its derivatives have been shown to have promising pharmacological activities, including hepatoprotective, anti-inflammatory, anti-oxidant and anti-cancer activities. Glycyrrhizic acid, a natural sweetener, has also been shown to possess multiple pharmacological activities (Ming and Yin, 2013).

Plant-origin bioactive ursane-type pentacyclic triterpenes include ursolic acid, corosolic acid, asiatic acid, weigelic acid, actinidic acid, madecassic acid etc. Ursolic acid has been isolated from several plant species, including herbs and fruits, such as Ocimum species and apple (Misra et al., 2014). Ursolic acid has been shown to have a wide spectrum of pharmacological activities including anti-cancer and anti-microbial activities (WoŸniak et al, 2015; Kashyap et al., 2016). Corosolic acid, a bioactive component of Lagerstroemia speciosa exhibits antihyperlipidemic, anti-inflammatory, anti-fungal and antimicrobial activities. The beneficial effects of corosolic acid, with respect to various aspects of glucose and lipid metabolism appear to be well studied (Stohs et al., 2012). Corosolic acid is considered as a potential drug for management of diabetes and its complications (Miura et al., 2012; Sivakumar et al., 2009). Taraxastane is a stereoisomer of ursane. Taraxasterol, a taraxastane-type triterpene isolated from medicinal herb Taraxacum officinale has several pharmacological activities, including anti-inflammatory and anti-cancer (Zhang et al., 2012; Sharma and Zafar, 2015).

Hydroxyhopanone, one of the constituents of dammar gum obtained from the Dipterocarpaceae family of trees, was the first known pentacyclic triterpene of hopane class. Its name was based on the genus Hopea, one of the sources of the dammar gum. However, hopanoids are found abundantly in bacteria as a component of cell membrane. Similarly, 
bacterial tetrahymanol is an example of pentacyclic triterpenenes with a $3 \beta-(21 \alpha-)$ hydroxy-substituted gammacerane structure. Pentacyclic triterpenes of serratane-type are common in herbs of the Huperziaceae family, such as Huperzia serrata, $H$. phlegmaria (Zhou et al., 2004; Shi et al., 2005). Friedelane-type pentacyclic triterpenes, such as friedelin and endodesmiadiol are common constituents of cork and these by-products of the cork processing industry are targeted for biovalorization (Moiteiro et al., 2006). Cycloartenol, the general sterol precursor in plants, is also classified under pentacyclic triterpene, although it is based on tetracyclic lanostane structure.

\section{Hexacyclic Triterpene}

Hexacyclic triterpenes are rarely found in nature (Fig. 1). Few hexacyclic triterpene acids namely, euscaphic acid A-F and a taraxerene-type hexacyclic triterpene acid have been isolated from Euscaphis japonica (Cheng et al., 2010 and Li et al., 2016). Among these, euscaphic acid $\mathrm{C}$ and $\mathrm{D}$ were found to be cytotoxic against different cancer cell lines. However, taraxerene-type hexacyclic triterpene showed promising inhibitory activity on oleic acid-induced triglyceride accumulation. Mudanpinoic acid A and kadlongilactones are other examples of hexacyclic triterpenes obtained from Paeonia suffructicosa and Kadsura longipedunculata, respectively (Lin et al., 1998; Pu et al., 2005). Kadlongilactone A and B exerted significant inhibitory effects against human tumor cells (Pu et al., 2005).

\section{Quassinoid and Tetranortriterpene}

Quassinoids are degraded triterpenes with $\mathrm{C}_{18}, \mathrm{C}_{19}$, $\mathrm{C}_{20}, \mathrm{C}_{22}$ and $\mathrm{C}_{25}$ skeletons. This class of triterpenes are mostly found in plants of the Simaroubaceae family. 15-O-acetylbruceolide is an example of quassinoid that was isolated from Brucea javanica and B. sumatrana (Fig. 1). Tetranortriterpenes are highly oxygenated triterpenes and have a prototypical structure, either containing or derived from a precursor with a 4,4,8-trimethyl-17-furanylsteroid skeleton (Fig. 1). Several tetranortriterpenes are isolated from neem (Azadirachta indica) and citrus have shown insecticidal activity (Roy and Saraf, 2006). Tetranortriterpenes are also known as limonoid based on the first isolated tetranortriterpenes, limonin from citrus.

\section{Saponin}

Saponins (derived from Latin word 'sapo', means 'soap') are glycosides containing one or more hydrophilic glycoside moieties combined with a lipophilic aglycone triterpene or steroid derivative (Sawai and Saito, 2011). Thus, saponins are classified as triterpene or steroidal saponin, based on the aglycone skeletons i.e. sapogenins (Fig. 1). Avenacin A-1, soyasaponins, asiaticosides, gypsosaponins and ginsenosides are the examples of triterpene saponins, whereas, asparasaponins, withalongolides, avenacosides and digoxin are the examples of steroidal saponins. Saponins are also recognised as hemolytic or non-hemolytic based on their hemolysis ability (Biazzi et al., 2015). Hemolytic activity is dependent on affinity of the saponin for the membrane sterols and is determined by the nature of aglycone sapogenin. Soyasapogenols-derived saponins with oxidation on C-24 position are devoid of hemolytic activity; however, hederagenin, zanhic acid and medicagenic acid-derived saponins with oxidation on C-28 position show hemolytic activity (Carelli et al., 2011). The oxidation on C-28 of triterpene moiety was found to be compatible with further oxidative modification on $\mathrm{C}-23$, but not on $\mathrm{C}-24$ position. These additional modifications on triterpene structure might have a role in determining the degree of hemolytic activity of the saponins. Similarly, oxidation on C-24 is compatible with additional modifications on C-21 and/or C-22 positions of the triterpenes. However, C-24 oxidation prevents oxidation on C-28 position (Tava et al., 2011).

Saponins have utilities as medicines, food additives and also as natural sweetener. As for example, gensinosides of Panax sp., withalongolides of Physalis longifolia, asparasaponins of Asparagus, vaccarosides of Saponaria vaccaria and glycyrrhizin of Glycyrrhiza sp. have potential pharmacological activities and are considered as the major bioactive components of the corresponding medicinal plants. Moreover, glycyrrhizin (150 times sweeter than sugar) is also used as a natural sweetener. Furthermore, saponins of Quillaja saponaria (soapbark) are useful as soap, and emulsifiers in cosmetics and foods (Sawai and Saito, 2011).

\section{Triterpene Biosynthetic Pathway}

All terpenes, including triterpenes are derived from 
isopentenyl diphosphate (IPP) and dimethylallyl diphosphate (DMAPP), two universal 5-carbon building blocks for the terpene biosynthesis (Garg et $a l .$, 2015). In fungi and animals, IPP and DMAPP are produced from acetyl-CoA via the mevalonic acid (MVA) pathway. In prokaryotes, IPP and DMAPP are produced from pyruvic acid and glyceraldehyde3-phosphate via the methylerythritol phosphate (MEP) pathway. In higher plants, both MVA and MEP pathways exist and these pathways operate independently in separate sub-cellular compartments. The cytoplasmic MVA pathway of plants is predominately responsible for the supply of IPP and DMAPP for the biosynthesis of sesquiterpenes (C15), triterpenes $(\mathrm{C} 30)$, and polyprenols $(>45)$. However, IPP and DMAPP derived from the plastidial MEP pathway are channelled for the biosynthesis of monoterpenes (C10), diterpenes (C20), sesterterpenes (C25), carotenoids (C40) and longchain phytol (Jiang et al., 2016; Liu et al., 2016). Two units of IPP and a DMAPP are joined to generate C15 farnesyl pyrophosphate (FPP) that serves as precursor for the biosynthesis of sesquiterpenes (Fig. 2). Two units of FPP are fused to generate the linear C30 triterpene squalene, the predominant triterpene precursor for the prokaryotes. However, squalene might also serve as a precursor for the biosynthesis of acyclic triterpenes in plants (Fig. 1). Epoxidation of squalene results in the formation of 2,3oxidosqualene which is the ubiquitous precursor for the biosynthesis of membrane sterol and diverse triterpenes in plants. Cyclization of 2,3-oxidosqualene to basic triterpene scaffolds is the first diversifying step in triterpene biosynthesis and also marks the branch point between the pathways for the biosynthesis of primary membrane sterols and steroid hormones, and secondary triterpene metabolites in plants. Cycloartenol, one of the pentacyclic triterpene skeletons formed from 2,3-oxidosqualene serves as a primary precursor for the sterols and steroid hormones in plants, whereas in case of fungi and animal, tetracyclic lanosterol serves the same purpose (Fig. 2). However, cyclization of 2,3-oxidosqualene to other triterpene skeletons described in the previous section initiates the formation of structurally diverse triterpenes in plants. Recently, plant genome mining also revealed the occurrence of enzymes in plants that can convert 2,3-oxidosqualene to lanosterol. These observations initiated a debate over consideration of cycloartenol as the sole precursor for sterol biosynthesis in plants (Ohyama et al., 2009). After initial cyclization, triterpene backbones undergo a plethora of modifications, like oxidation, methylation, acetylation, malonylation and glycosylation, catalysed by CYP450s, ATs and GTs, to form bioactive compounds (Osbourn et al., 2011).

\section{Biological Role and Spatio-Temporal Accumulation Pattern of Triterpenes}

Sterols and steroid hormones play basic function in plants and thus, are considered as primary metabolites. Phytosterols such as campesterol, $\beta$-sitosterol and stigmasterol are the vital structural components of the cell membranes. It has been shown that impaired phytosterol biosynthesis affects plant growth and development (Gas-Pascual et al., 2014). Similarly, the ability of the plants to biosynthesize and, also to perceive and respond to steroidal hormones brassinosteroids (BRs) is essential to normal growth and development (Haubrick and Assmann, 2006). In contrast, triterpene secondary metabolites are not generally regarded as essential for normal growth and development of the plants; although, the biological roles of most of them are yet to be determined. The insecticidal and antimicrobial activities of several triterpenes led to a speculation of general protective role of triterpenes against pests and pathogens (González-Coloma et al., 2011; Kurek et al., 2012; Misra et al., 2014). The first direct evidence for the role of triterpene saponin in plant defence arose from the genetic analysis of saponin-deficient ( $\mathrm{sad}$ ) mutants of oat (Avena strigosa) (Papadopoulou et al., 1999). In oat, antimicrobial triterpene saponins, known as avenacins, accumulate constitutively in roots. sad mutants were deficient in biosynthesis of root avenacins and were compromised in their resistance to a variety of root-infecting fungal pathogens, including Gaemannomyces graminis, Fusarium culmorum and $F$. avenaceum (Papadopoulou et al., 1999). However, sad mutants were not susceptible to leaf-infecting fungal pathogens, suggesting the spatio-temporal role of avenacins in mediating disease resistance. Similarly, tissue- and organ-specific accumulation of saponins in other plant species was also reported. For example, glycyrrhizin and ginsenosides, in Glycyrrhiza sp. and Panax sp., respectively, accumulate in roots (Shan et al., 2001; Fukuda et al., 2006). Consistent with the root-specific 
accumulation of saponins, biosynthetic pathway genes also express specifically in roots (Mylona et al., 2008; Mugford et al., 2009).

Interestingly, one of the sad mutants ( $\operatorname{sad2}$ ) of oat accumulates abnormally high levels of pentacyclic triterpene $\beta$-amyrin, an intermediate for the avenacin biosynthetic pathway. Elevated level of $\beta$-amyrin in sad2 mutants triggers a "superhairy" root phenotype, because of transformation of a greater proportion of epidermal cells into root hair cells, rather than nonhair cells (Kemen et al., 2014). These findings provided direct evidence that abnormal accumulation of a triterpene can affect root development. Similarly, a role for lupeol biosynthesis in Lotus japonicas nodule formation was proposed (Delis et al., 2011). The gene (OSC3) involved in lupeol biosynthesis was found to exclusively express in roots and nodules, and silencing of $\mathrm{OSC} 3$ resulted in a more rapid nodulation phenotype. Furthermore, genetic and physiological analysis of A. thaliana knock-out mutants ( $m r n l)$ that are unable to biosynthesize monocyclic triterpene marneral, revealed the critical role of marneral-derived triterpene(s) in plant growth and development (Go et $a l ., 2012)$. mrnl mutants were affected in the level of triterpenes and sterols, and in cell division and elongation process. $\mathrm{mrnl}$ mutants displayed abnormal shoot and root phenotypes, late flowering, and delayed embryogenesis as compared to the wild type counterpart. The gene responsible for marneral biosynthesis (MRN1) was found to specifically express in shoot and root apical meristems.

Triterpenes are biosynthesized by plants often in response to biotic and abiotic stress, and also in response to treatment with methyl jasmonate (MeJA), a phytohormone that mediates stress responses in plants. Similarly, the expression of triterpene biosynthetic pathway genes is induced when plants are subjected to stressful conditions or applied with MeJA (Suzuki et al., 2002; Phillips et al., 2006; Misra et al., 2014; Moses et al., 2015a,b). Furthermore, triterpenes, particularly pentacyclic triterpenes such as ursolic acid, oleanolic acid and amyrones accumulate in cuticle of plant surface, and the genes involved in their biosynthesis express in epidermal cells or in filamentous trichomes (Murata et al., 2008; Misra et al., 2014; Moses et al., 2015b). However, vacuole is considered as the sub-cellular location for saponins because of their increased polarity compared to the aglycone triterpenes (Mylona et al., 2008). Because cuticle is considered as protective barrier against pathogens and water loss (Yeats and Rose, 2013), accumulation of triterpenes in cuticle signifies their role in plant defense against biotic and abiotic stress. These observations also suggested the differences in the site of biosynthesis and storage for the triterpenes, and possible involvement of transporter(s) for the secretion of these triterpenes into cuticle from epidermal cells or filamentous trichomes (Misra et al., 2014). The accumulation of ursolic acid and oleanolic acid was found to be tissuespecific and developmentally regulated, and also responsive to MeJA treatment in plants (Yu et al., 2013; Misra et al., 2014). Moreover, the biosynthetic pathway genes also show spatio-temporal expression pattern in plants. In Arabidopsis and oat, the biosynthetic pathways for triterpenes thalianol/ marneral, and avenacins, respectively, have been shown to be organized as metabolic gene clusters (Field and Osbourn 2008; Field et al., 2011; Qi et al., 2004; Mugford et al., 2013). Clustering of genes is likely to facilitate co-inheritance of the biosynthetic pathway and co-regulation of the genes at the level of chromatin (Nützmann and Osbourn, 2014).

\section{Oxidosqualene Cyclase: Catalyzing the Initial Diversifying Step in Triterpene Biosynthesis}

2,3-Oxidosqualene is a common biosynthetic intermediate for plant triterpenes, sterols and steroid hormones (Fig. 2). The initial diversifying step in triterpene/sterol pathway is catalyzed by a family of enzymes, known as oxidosqualene cyclases (OSC), that can convert 2,3-oxidosqualene into a variety of cyclic triterpenes. A. thaliana cycloartenol synthase (AtCAS1) was the first OSC to be cloned from a plant species (Corey et al., 1993). AtCAS1 was identified while screening for CAS activity by transforming an A. thaliana cDNA expression library in a yeast (Saccharomyces cerevisiae) mutant background that accumulates 2,3-oxidosqualene because of mutation in a lanosterol synthase gene $(L A S)$. LAS, an OSC, is required for the biosynthesis of lanosterol, an ergosterol precursor in yeast and other fungi. The cyclization of 2,3-oxidosqualene into cycloartenol by the CAS is considered as the first committed step and also as the major contributor for the biosynthesis of essential phytosterols (e.g. campesterol, $\beta$-sitosterol and stigmasterol) and steroid 


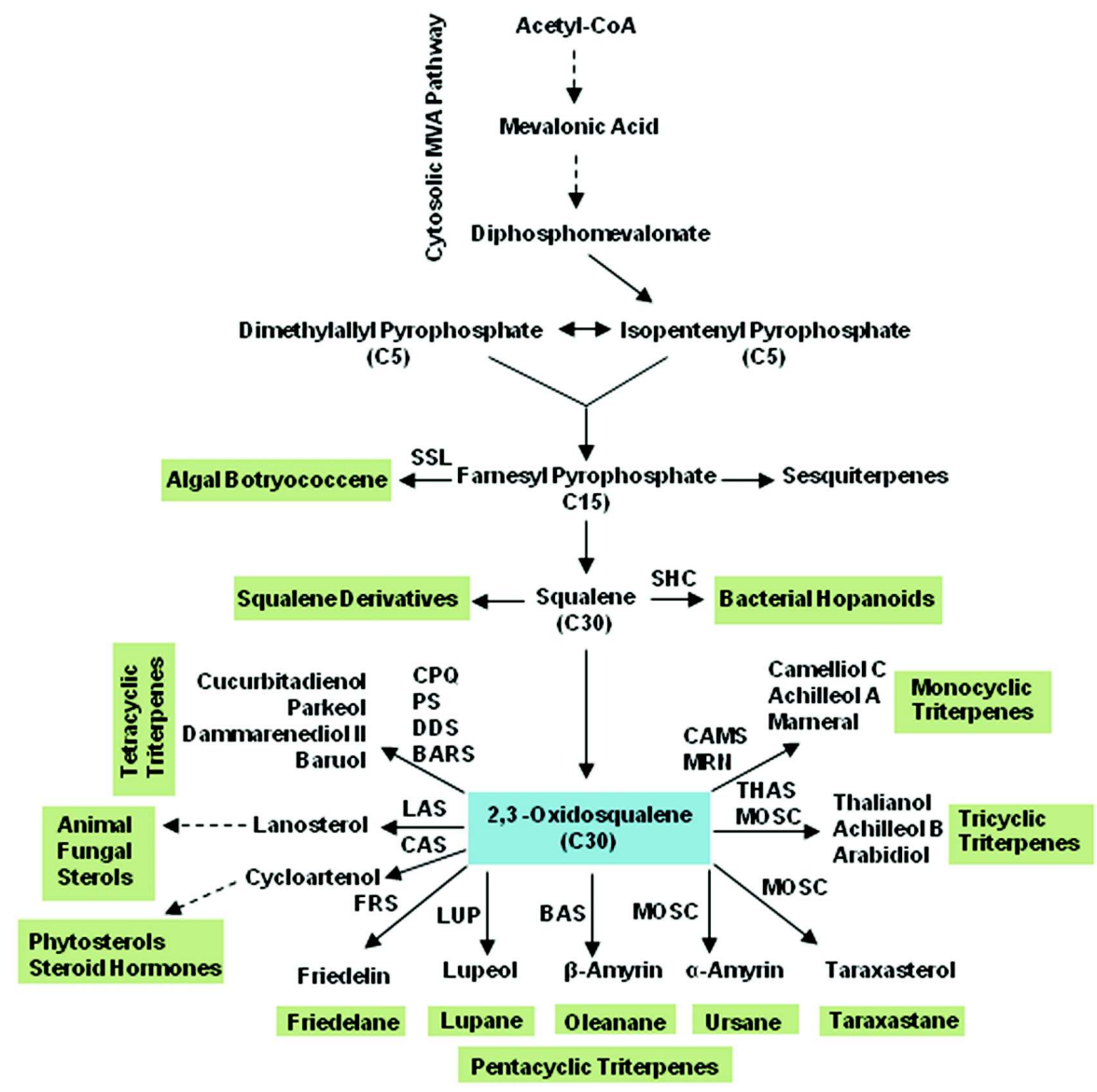

Fig. 2: Triterpene biosynthetic pathway. Triterpenes produced from farnesyl pyrophosphate, squalene and 2,3-oxidosqualene are shown. Oxidosqualene cyclase-mediated conversion of 2,3-oxidosqualene into diverse triterpene skeletons is highlighted. Oxidosqualene cyclases are abbreviated as CPQ (cucurbitadienol synthase), PS (parkeol synthase), DDS (dammarenediol II synthase), BARS (baruol synthase), LSS (lanosterol synthase), CAS (cycloartenol synthase), FRS (friedelin synthase), LUP (lupeol synthase), BAS ( $\beta$-amyrin synthase), MOSC (multi-functional oxidosqualene cyclase), THAS (thalianol synthase), MRN (marneral synthase) and CAMS (camelliol C synthase). SSL and SHC denote squalene synthase-like and squalene hopene cyclase, respectively

hormones (BRs) in plants. The recent identification of LAS in plants also suggested a possible role of lanosterol as sterol/triterpene precursor in plants (Suzuki et al., 2006; Ohyama et al., 2009); although, the contribution of LAS towards major phytosterol biosynthesis and physiology of the plants might be minor (Gas-Pascual et al., 2014). After the identification of AtCAS1, several other OSCs were cloned from plants, mostly following homology-based mining of the sequence databases and Polymerase Chain Reaction (PCR) using degenerate primers. These efforts led to the discovery of several divergent plant OSCs that can convert 2,3-oxidosqualene to products other than cycloartenol such as amyrins, lupeol, cucurbitadienol, marneral, etc. (Fig. 2). In the following sections, plant OSCs will be discussed with respect to their product specificities and reaction mechanisms.

\section{Mono-functional and Multi-functional Oxidosqualene Cyclases}

With the availability of genomic and transcriptomic sequences, a number of plant OSCs are now being identified and characterized for their product specificities, mostly based on enzyme expression on heterologous hosts. To date, nearly 90 OSCs from plants are biochemically characterized for their product specificities (Table 1). The occurrence of several homologues in a single plant species suggests that OSCs are encoded by multi-gene family and thus, a 
single plant is able to produce multiple triterpene scaffolds. For example, A. thaliana and $O$. sativa genomes encode 13 and 9 OSCs, respectively. All the OSCs of A. thaliana are studied for their product specificities, however, enzymatic characterization of four of the $O$. sativa OSCs is completed so far (Table 1). Five of the A. thaliana OSCs are mono-functional and catalyze the cyclization of 2,3-oxidosqualene into either of the cyclic triterpenes, cycloartenol, lanosterol, $\beta$-amyrin, marneral and thalianol. However, rest of the Arabidopsis OSCs are multi-functional in nature and are able to convert 2,3-oxidosqualene into multiple cyclic triterpenes that can range from 2 to 23 in number, with at least one major triterpene product such as baruol, camelliol C, 33,20-dihydroxylupane, $\beta$-amyrin, tirucalla-7,21-dien-33-ol, arabidiol, tirucalla-7,24-dien3beta-ol, lupeol. Among the characterized rice OSCs, three are mono-functional and convert 2,3oxidosqualene into either of cycloartenol, parkeol and isoarborinol. However, the fourth one produces achilleol B as major product along with few other minor products (Table 1).

After identification of the first multifunctional OSC (LUP1) from Arabidopsis (Segura et al., 2000), a number of multi-functional OSCs are being isolated and characterized from plants. It is now well recognised that the diversity of plant triterpenes originates not only from the occurrence of the diverse monofunctional OSCs but also from the ability of some OSCs to produce multiple products (Table 1). Recently, our laboratory has reported identification and characterization of two OSCs (ObAS1 and ObAS2) from $O$. basilicum (Misra et al., 2014). Heterologous expression of these OSCs in $S$. cerevisiae revealed their identity as $\beta$-amyrin synthase (ObAS1) and mixed amyrin synthase, with a product preference for $\alpha$-amyrin (ObAS2). Product specificities of ObAS1 and ObAS2 suggested that their major roles are to provide precursors for the biosynthesis of oleanane-type and ursane-type pentacyclic triterpenes, respectively. Multifunctionalities of OSCs have possibly arisen repeatedly in plant kingdom during evolution. Moreover, multifunctional nature of an OSC might reflect evolution of a mono-functional enzyme in progress. Directed evolution and mutagenesis studies have revealed that minor changes in $O S C$ sequences can lead to product diversity (Segura et al., 2003).
A list of the mono-functional and multifunctional plant OSCs are provided in the Table 1 . The presence of mono-functional CAS in all examined plant lineages, including angiosperm, gymnosperm, pteridophyte, and primary function of cycloartenol-derived sterols and steroid hormones in plants (Gas-Pascual et al., 2014), suggest that CAS has evolved for the purpose of primary function in plant kingdom. Moreover, the similarity of plant CASs with amoebae and bacteria CASs suggests that CAS activity possibility originated before the emergence of plant species (Phillips et al., 2006). Although, $\beta$-amyrin synthase is widely distributed among angiosperms, it is not so far identified in gymnosperms and lower plants (Table 1). OSCs that have limited species distribution, had possibly originated from CAS following gene duplication and neo-functionalization to perform secondary functions in plants. The high degree of amino acid identities among OSCs of different plant species with diverse product specificities, further supports this view (Fig. 3 ). The mono-functional LAS which provide general sterol precursor lanosterol for animals, fungi, and trypanosomatids, is also found in few plant species (Table 1). The presence of both LAS and CAS in plants suggests that plants need some cycloartenolderived metabolites that cannot be produced from lanosterol. Arabidopsis LAS is $65 \%$ identical with the AtCAS1 at the amino acid level (Fig. 3). Interestingly, AtCAS1 can be engineered to an accurate LAS following only two amino-acid substitutions (H477N/ I481V), suggesting that only a small change in the DNA sequence could transform CAS to LAS during enzyme evolution (Lodeiro et al., 2005).

Although, the identified OSCs will be useful in enhancing triterpene yield in native plants and also in heterologous systems, the major challenge is engineering of a multi-functional OSC to a more accurate mono-functional OSC. In this way, it will be possible to reduce chemical complexity of triterpene extracts in engineering plants or in other hosts. For example, ObAS1 is a mono-functional $\beta$-amyrin synthase. Thus, expression of ObAS1 in S. cerevisiae led to formation of $\beta$-amyrin. However, ObAS2 is a multi-functional OSC with a product preference for $\alpha$-amyrin. Therefore, expression of ObAS2 in $S$. cerevisiae led to formation of both $\alpha$-amyrin and $\beta$ amyrin (Misra et al., 2014). Since, these amyrins are structural isomers, their separation will require complex extraction and isolation protocol. It will be 
Table 1. Product specificity of plant oxidosqualene cyclases

\begin{tabular}{|c|c|c|}
\hline $\begin{array}{l}\text { Product(s) of the oxidosqualene cyclase- } \\
\text { catalyzed reaction }\end{array}$ & $\begin{array}{l}\text { Product(s) } \\
\text { flux into } \\
\text { major triter- } \\
\text { pene pathway }\end{array}$ & Source Plants* \\
\hline Cycloartenol & Phytosterol & $\begin{array}{l}\text { Abies magnifica (AF216755), Adiantum capillus-veneris (AB368375), } \\
\text { Arabidopsis thaliana (At2g07050), Artemisia annua (KM670093), Betula } \\
\text { platyphylla (AB055509), Chlamydomonas reinhardtii (EDP09612), Costus } \\
\text { speciosus (AB058507), Cucurbita pepo (AB116237), Dioscorea } \\
\text { zingiberensis (AM697885), Glycyrrhiza glabra (AB025968), Kalanchoe } \\
\text { daigremontiana (HM623872), Kandelia candel (AB292609), Lotus } \\
\text { japonicas (AB181246), Luffa cylindrical (AB033334), Oryza sativa } \\
\text { (AK121211), Panax ginseng (AB009029), Pisum sativum (D89619), } \\
\text { Polypodiodes niponica (AB530328), Rhizophora stylosa (AB292608), } \\
\text { Ricinus communis (DQ268870), Withania somnifera (HM037907) }\end{array}$ \\
\hline Lanosterol & Phytosterol & A. thaliana (At3g45130), L. japonicas (AB244671), P. ginseng (AB009031) \\
\hline Lupeol & Pentacyclic & $\begin{array}{l}\text { B. platyphylla (AB055511), Bruguiera gymnorrhiza (AB289586), G. glabra } \\
\text { (AB116228), L. japonicas (AB181245), Olea europaea (AB025343), } R \text {. } \\
\text { communis (DQ268869), Taraxacum officinale (AB025345), W. somnifera } \\
\text { (JQ728552) }\end{array}$ \\
\hline$\beta$-Amyrin & Pentacyclic & $\begin{array}{l}\text { A. thaliana (At1g78950), A. annua (EU330197), Aster sedifolius (AY836006), } \\
\text { Avena strigosa (AJ311789), B. platyphyllai (AB055512), B. gymnorrhiza } \\
\text { (AB289585), Euphorbia tirucalli (AB206469), Gentiana straminea } \\
\text { (FJ790411), G. glabra (AB037203), L. japonicas (AB181244), Medicago } \\
\text { truncatula (AJ430607), Maesa lanceolata (KF425519), Nigella sativa } \\
\text { (FJ013228), Ocimum basilicum (KF636411), P. ginseng (AB009030), P. } \\
\text { sativum (AB034802), Polygala tenuifolia (EF107623), Saponaria vaccaria } \\
\text { (DQ915167), Solanum lycopersicum (HQ266579), W. somnifera } \\
\text { (JQ728553) }\end{array}$ \\
\hline Cucurbitadienol & Tetracyclic & C. pepo(AB116238) \\
\hline Marneral & Monocyclic & A. thaliana (At5g42600) \\
\hline Thalianol & Tricyclic & A. thaliana (At5g48010) \\
\hline Parkeol & Tetracyclic & O. sativa (AK066327) \\
\hline Isomultiflorenol & Pentacyclic & L. cylindrical (AB058643) \\
\hline Isoarborinol & Pentacyclic & O. sativa (AK067451) \\
\hline Dammarenediol II & Tetracyclic & P. ginseng (AB265170), Centella asiatica (AY520818) \\
\hline Baccharis oxide & Pentacyclic & Stevia rebaudiana (AB455264) \\
\hline $\begin{array}{l}\alpha \text {-amyrin (major), } \beta \text {-amyrin, } \\
\psi \text {-taraxasterol, and butyrospermol }\end{array}$ & Pentacyclic & O. europaea (AB291240) \\
\hline$\alpha$-Amyrin and $\beta$-amyrin in 5:3 ratio & Pentacyclic & O. basilicum (JQ809437) \\
\hline $\begin{array}{l}\text { Baruol }(89.7 \%) \text { along with } 22 \text { minor } \\
\text { products }\end{array}$ & Tetracyclic & A. thaliana (At4g15370) \\
\hline $\begin{array}{l}\text { Camelliol C }(98 \%) \text {, achilleol A }(2 \%) \\
\text { and } \beta \text {-amyrin }(0.2 \%)\end{array}$ & Monocyclic & A. thaliana (At1g78955) \\
\hline $\begin{array}{l}\text { 3 } \beta, 20 \text {-dihydroxylupane (major), } \beta \text {-amyrin, } \\
\text { germanicol, taraxasterol, } \psi \text {-taraxasterol }\end{array}$ & Pentacyclic & A. thaliana (At1g78970) \\
\hline $\begin{array}{l}\beta \text {-amyrin and taraxasterol (major) along } \\
\text { with at least } 7 \text { minor products }\end{array}$ & Pentacyclic & A. thaliana (At1g78960) \\
\hline $\begin{array}{l}\text { Tirucalla-7,21-dien-3 } 3 \text {-ol and additional } \\
\text { products }\end{array}$ & Tetracyclic & A. thaliana (At1g66960) \\
\hline
\end{tabular}


Arabidiol (major) and arabidiol 20,21epoxide

Tirucalla-7,24-dien-3 $\beta$-ol (85\%) and five minor products including butyrospermol (6\%), tirucallol (6\%), isotirucallol (1.5\%), 13ßH-malabarica-14(27),21-trien-3-ol (1\%), dammara-20,24-dien-33-ol (0.5\%)

Lupeol, $\alpha$-amyrin, bauerenol, $\alpha$ - and $\beta$-seco-amyrin

$\beta$-Amyrin, germanicol, lupeol and additional uncharacterized products

Lupeol, $\beta$-amyrin and $\alpha$-amyrin in 2:1:1

ratio

$\beta$-amyrin and lupeol in approximately equal amounts, and additional minor products

Achilleol B (90\%), tetracyclic (5.12\%) and pentacyclic scaffolds $(4.37 \%)$, and unidentified triterpenes $(0.51 \%)$

$\alpha$-amyrin and $\beta$-amyrin in 3:2 ratio along with at latest six minor products

Germanicol, $\beta$-amyrin and lupeol in the ratio of $63: 33: 4$

Taraxerol, $\beta$-amyrin and lupeol in the proportions $70: 17: 13$

$\delta$-amyrin (48\%), $\alpha$-amyrin (18\%), $\beta$-amyrin Pentacyclic $(13 \%)$ and four additional minor products including multiflorenol, $\psi$-taraxasterol, taraxasterol

$\alpha$-amyrin (major), $\beta$-amyrin and $\delta$-amyrin $\quad$ Pentacyclic in 5.5:2.7:1.8 ratio

Lupeol and additional uncharacterized products

$\alpha$-Amyrin (major) and $\beta$-amyrin

Lupeol (93\%), $\beta$-amyrin (7\%)

Taraxerol (60\%), $\beta$-amyrin (40\%)

Glutinol (67\%), friedelin (14\%), $\beta$-amyrin (11\%), taraxerol (8\%)

Friedelin (72\%), $\beta$-amyrin (18\%), taraxerol (10\%)

Shionone (90\%), $\beta$-amyrin (2\%), friedelin (2\%), dammara-20,24-dienol (1.5\%),

4-epishionone (1.5\%)

$\alpha$-Amyrin and $\beta$-amyrin in 5:1 ratio
Tricyclic

Tetracyclic

A. thaliana (At4g15340)

A. thaliana (At5g36150)

Pentacyclic

A. thaliana (At1g78500)

Pentacyclic

C. speciosus (AB058508)

Pentacyclic

K. candel (AB257507)

Pentacyclic

L. japonicas (AF478455)

Tricyclic

O. sativa (AK070534)

Pentacyclic

P. sativum (AB034803)

Pentacyclic

R. stylosa (AB263203)

Pentacyclic

R. stylosa (AB263204)

S. lycopersicum (HQ266580)

A. апnиа (KF309252)

Pentacyclic

A. annua (KM670094)

Pentacyclic

Catharanthus roseus (AFJ19235)

Pentacyclic

K. daigremontiana (HM623871)

Pentacyclic

K. daigremontiana (HM623868)

Pentacyclic

K. daigremontiana (HM623869)

Pentacyclic

K. daigremontiana (HM623870)

Tetracyclic

A. tataricus (AB609123)

Pentacyclic

Malus domestica (FJ032006)

*Source plants for the identified oxidosqualene cyclases along with GenBank/TAIR ID are mentioned.

quite helpful if the multi-functional ObAS2 can be engineered to a precise mono-functional $\alpha$-amyrin synthase. For this purpose, understanding of the structural and biochemical basis of OSC-mediated conversion of 2,3-oxidosqualene into cyclic triterpenes is essential. 


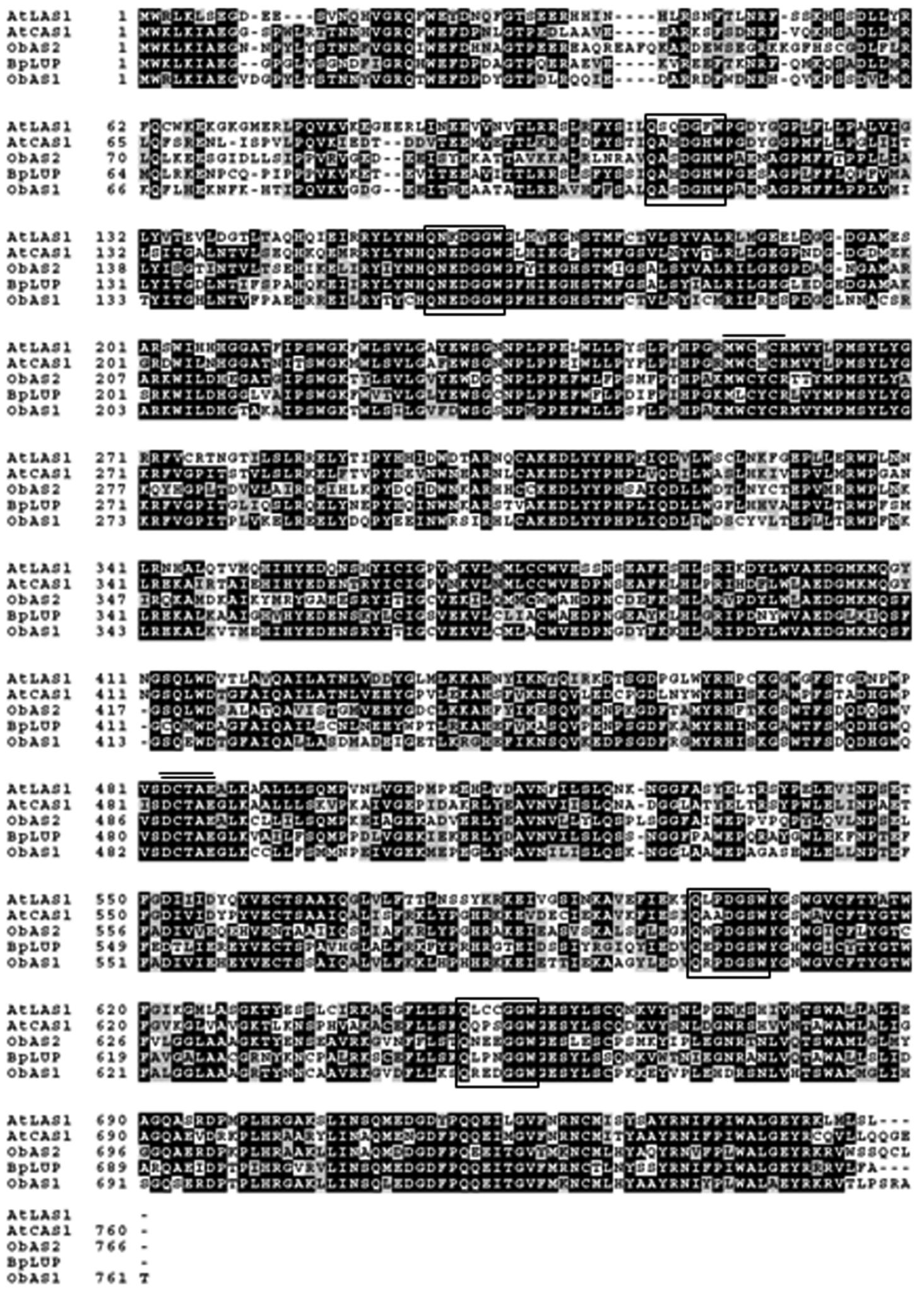

Fig. 3: Sequence alignment of some oxidosqualene cyclases commonly distributed among plant species. Arabidopsis cycloartenol synthase (AtCAS1, AT2G07050) and lanosterol synthase (AtLAS1, AT3G45130), Betula platyphylla lupeol synthase (BpLUP, BAB83087), $O$. basilicum $\beta$-amyrin synthase (ObAS1, KF636411) and mixed amyrin synthase (ObAS2, JQ809437) are the representative OSCs. QW, MWCYCR and DCTAE motifs are marked with boxes, single line and double line, respectively. Multiple sequence alignment and shading of amino acids were carried out following CLUSTAL Omega (http://www.ebi.ac.uk/) and BOXSHADE version 3.21 (http://www.ch.embnet.org/) 


\section{Structural and Biochemical Basis of Oxidosqualene Cyclase Catalyzed Reactions}

The study of OSC-mediated cyclization reaction has attracted organic chemists and biochemists for more than past fifty years. The conversion of 2,3oxidosqualene into cyclic triterpenes is considered as one of the most complex enzymatic reactions that occur in terpene metabolism (Thimmappa et al., 2014). Current understanding on biochemical basis of OSC-catalyzed reactions suggests that cyclization of 2,3-oxidosqualene involves a cascade of processes that start with substrate binding to the enzyme active site and proper folding of the substrate. This is followed by protonation of epoxide of 2,3-oxidosqualene by the enzyme which initiates a cascade of cyclization and rearrangement of carbocation intermediates. The reaction is finally terminated by either deprotonation or addition of water molecule to a carbocation intermediate to yield triterpene product (Abe, 2007). Variation in substrate folding, and carbocation cyclization and rearrangement steps contribute to triterpenes scaffold diversity (Figs. 4 and 5). The initial substrate folding step is crucial in determining which of the cyclization pathways to be followed. Folding of substrate into Chair-Boat-Chair (CBC) conformation directs a cyclization reaction for the formation of the protosteryl cation that generally gives rise to sterol precursors, cycloartenol and lanosterol, although some triterpene precursors such as parkeol and cucurbitadienol are also produced. However, ChairChair-Chair (CCC) conformation leads to the dammarenyl cation for the formation of more diverse triterpene skeletons based on further variation in carbocation cyclization and rearrangement steps (Figs. 4 and 5).

Unlike class I terpene synthases such as monoterpene, sesquiterpene, and class I diterpene (e.g. kaurene) synthases that catalyze ionizationinitiated cyclization with the help of a divalent cation $\left(\mathrm{Mg}^{2+}\right.$ or $\left.\mathrm{Mn}^{2+}\right)$ as cofactor, OSCs are classified as class II terpene synthases, similar to class II diterpene synthases such as copalyl diphosphate synthase, which catalyzes protonation-initiated cyclization (Misra et al., 2015). The roles of OSCs during 2,3oxidosqualene cyclization are well recognized as (I) a catalyst that presents a general acid of sufficient strength to protonate epoxide of 2,3-oxidosqualene and thereby, initiates sequential cyclization and rearrangement steps, (II) a chaperone that guides flexible substrate and highly reactive carbocation intermediates through a sequence of accurate conformational changes for the formation of correct cyclization product, (III), a shield that protects carbocation intermediates from premature deprotonation or addition of water, and (IV) provides an electron-rich environment that stabilizes proper carbocation intermediate, through cation- $\pi$ interactions, for promoting carbon-carbon bond forming reactions (Abe, 2007). Thus, mono-functional OSCs present their precise control over cyclization and rearrangement steps towards a single triterpene product. However, multi-functional OSCs possibly reflect their failure to precisely control cyclization and rearrangement steps for a particular triterpene product type. As for example, the differential product specificities of $O$. basilicum amyrin synthases (ObAS1 and ObAS2) are discussed (Fig. 5; Misra et al., 2014). The differential stability of the $\mathrm{C}-19$ oleanyl and $\mathrm{C}-13$ ursanyl carbocations bound to the enzyme is likely to affect the product specificities of these OSCs. The precise control over the hydride shifts and deprotonation of the C-19 oleanyl carbocation by ObAS1 results in the formation of only $\beta$-amyrin. However, ObAS2 catalyzes the formation of more $\alpha$-amyrin than $\beta$-amyrin; therefore, it might favour the formation of the ursane-type over the oleananetype pentacyclic triterpene by directing the reaction towards C-13 ursanyl carbocation following a C-20 methyl shift, instead of completely stabilizing the C19 oleanyl carbocation (Morita et al., 2000; Abe et al., 2007; Misra et al., 2014).

A well known example of multi-functionality in OSC family is provided by the Arabidopsis BARS1 that makes tetracyclic baruol as a major cyclization product; however, it also produces 22 additional triterpenes as minor products (Lodeiro et al., 2007). The number of products obtained from BARS1catalyzed reaction is the highest for any known mulifunctional OSCs. BARS1 catalyzes formation of diverse triterpene skeletons including monocycles, tricycles, tetracycles and pentacycles. Thus, it was proposed that BARS1 is able to deprotonate at 14 distinct sites, ranging from A to E ring of the triterpene skeleton. Therefore, multi-functionality of BARS1 might be due to its failure to provide a tight control over 2,3-oxidosqualene cyclization towards baruol. 'Negative catalysis', a concept that was proposed to 


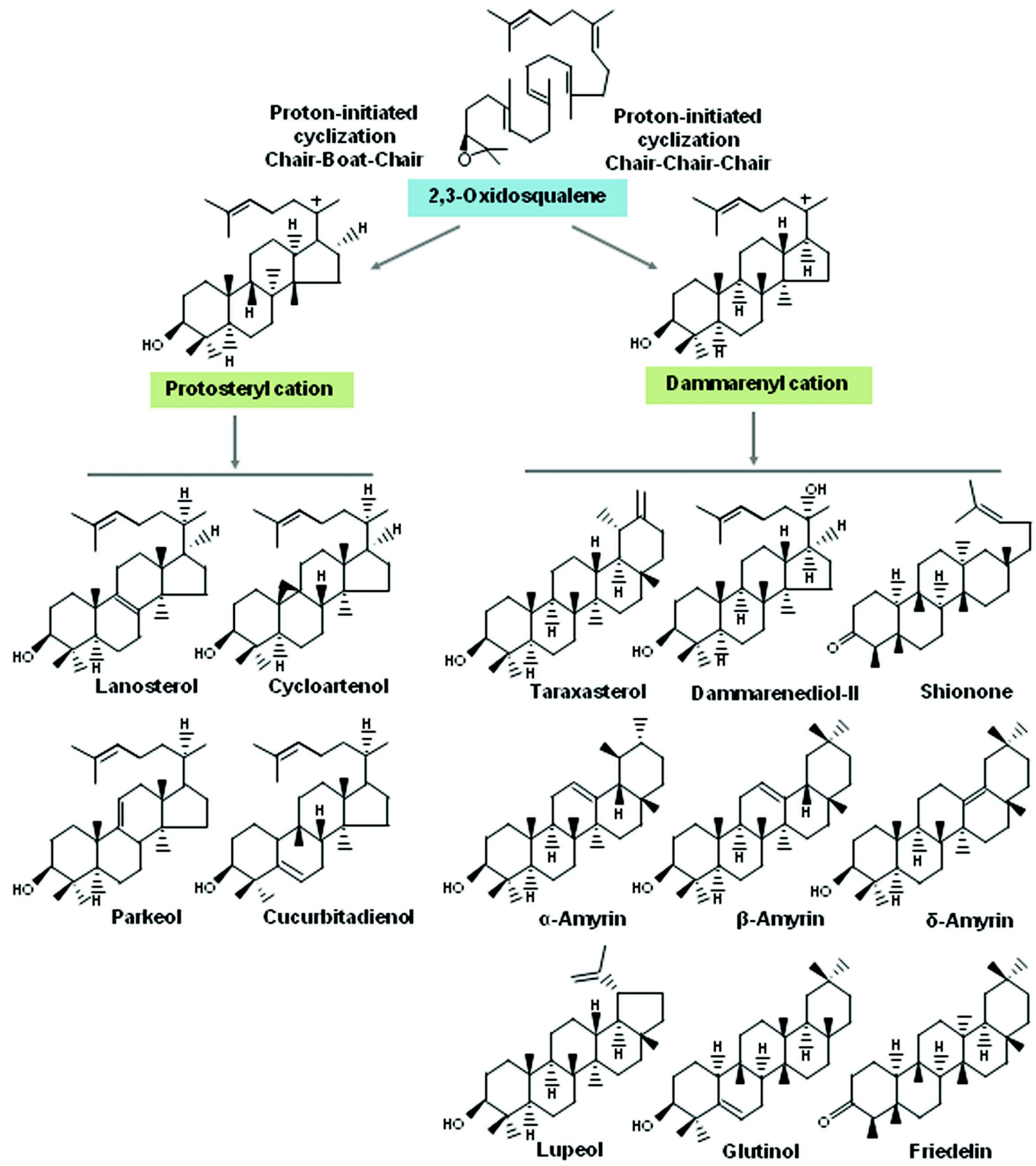

Fig. 4: Oxidosqualene cyclase-mediated cyclization of 2,3-oxidosqualene to different triterpenes. Folding of 2,3-oxidosqualene into Chair-Boat-Chair (CBC) or Chair-Chair-Chair (CCC) conformation leads to a protonation-initiated cyclization reaction for the formation of protosteryl or dammarenyl cation, respectively. Based on variation in cyclization and rearrangement of carbocations, diverse triterpene skeletons are formed

explain the mechanism by which enzymes deal with highly reactive intermediates, seems to fit very well with the mechanism of OSC-catalyzed reaction (Retey, 1990). According to this concept, the actual role of an OSC might be to block alternative cyclization paths, instead of stabilizing a reaction intermediate, that is being directed towards a particular product. Therefore, product diversity might be the default for 


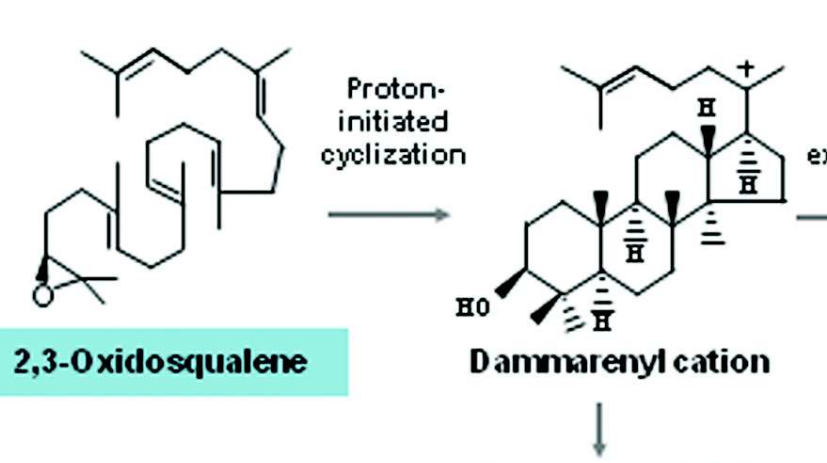

Dammarenecliol-II

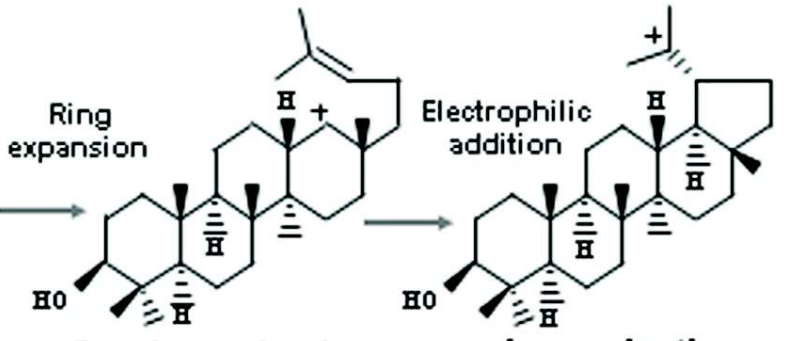

Baccharenyl cation

Lupenyl cation

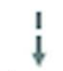

Shionone

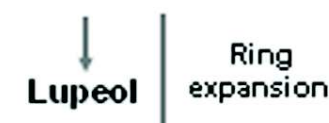

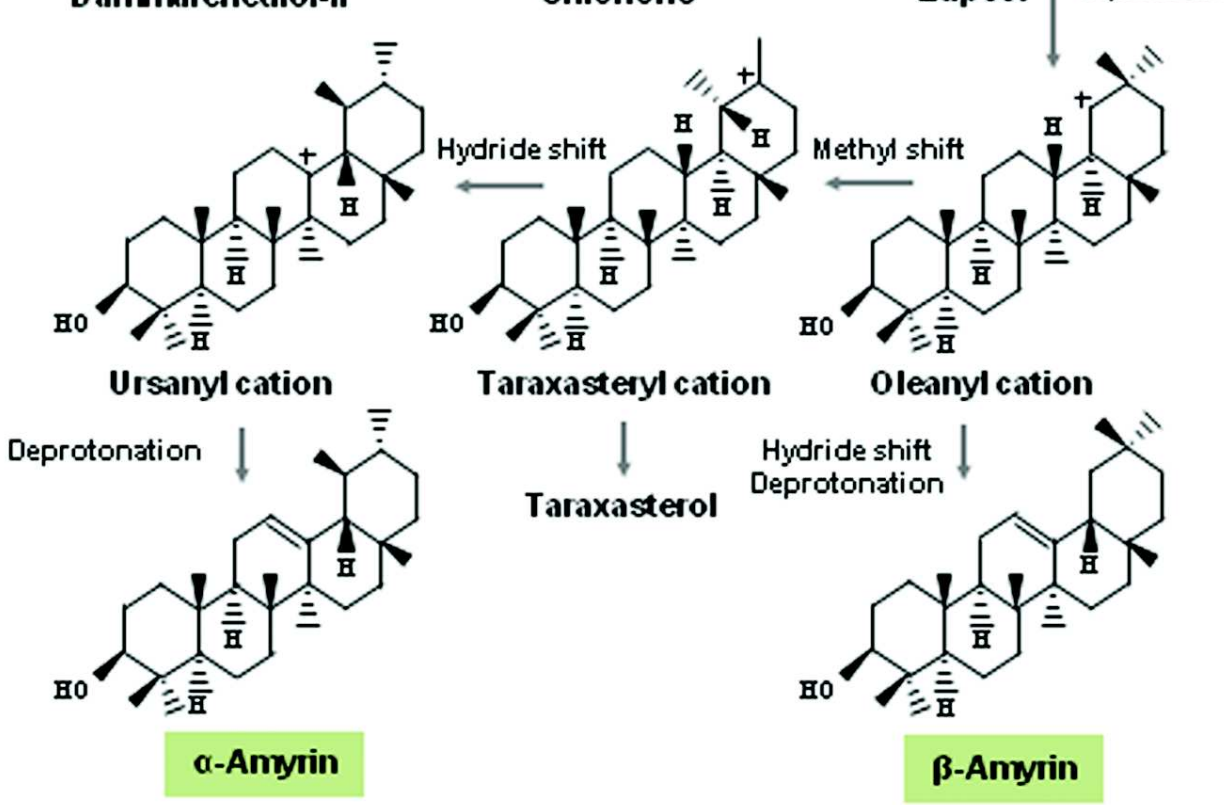

Fig. 5: Oxidosqualene cyclase-mediated cyclization of 2,3-oxidosqualene to ursane-type ( $\alpha$-amyrin) and oleanane-type $(\beta$ amyrin) pentacyclic triterpenes. Different carbocation intermediates and their routes for the formation of alternate triterpenes (e.g. dammarenediol-II, shionone, lupeol and taraxasterol) are shown

OSCs, considering the mechanism of 'negative catalysis' and however, the specificity for a particular product type might be due to the exclusion of alternative pathways (Lodeiro et al., 2007).

Although, crystal structure of plant OSC is yet to be determined, crystal structures of a human OSC (LAS) and an Alicyclobacillus acidocaldarius squalene hopene cyclase (SHC) which catalyzes conversion of squalene to hopene, are resolved (Wendt et al., 1997, 1999; Thoma et al., 2004). These crystal structures provided important structural insights into OSC-catalyzed reaction. Human LAS and $A$. acidocaldarius SHC utilize different substrates (2,3oxidosqualene and squalene, respectively) and share only about $25 \%$ amino acid sequence identity, however, they have very similar structural architectures, including two highly conserved $(\alpha \alpha)$ barrel domains (domain I and II) and a hydrophobic membraneinsertion helix. Domain I and II connect a large active site cavity present at the centre of the enzyme. A channel is led to the active site cavity from the membrane-inserted region (Thoma et al., 2004). The central section of the cavity is lined with a chain of conserved aromatic amino acids that possibly function in stabilizing the carbocation intermediates through cation- $\pi$ interactions. However, the top and bottom regions of the cavity are formed by polar hydrogenbonding networks around the charged amino acids, and function in the initial substrate protonation and in the final carbocation deprotonation, respectively (Abe et al., 2007). OSCs and SHC are monotopic 
membrane proteins without any protruding through the phospholipid bilayer. Lipophilic substrate enters the enzyme active site cavity through membrane and product is released from the active site cavity to the membrane (Reinert et al., 2004; Thoma et al., 2004). Directed evolution and site-directed mutational studies on OSCs and SHC led to identification of several conserved motifs and amino acids that are important for protonation of substrate, cyclization, rearrangement and deprotonation of carbocation intermediates (Kushiro et al., 1999; Hart et al., 1999; Dang and Prestwich, 2000; Joubert et al., 2000; Herrera et al., 2000; Kushiro et al., 2000; Segura et al., 2003; Lodeiro et al., 2004; Lodeiro et al., 2005; Chang et al., 2013). OSCs have a conserved catalytic motif DCTAEA in which aspartic acid serves as general acid that protonates the substrate (Fig. 3). In case of SHC, DDTAVV motif performs the same function. Interestingly, a SHC mutant in which DDTAVV motif was changed to DCTAEA was unable to accept squalene as substrate; however, the mutant protein was still able to catalyze cyclization of 2,3oxidosqualene (Dang and Prestwich, 2000). MWCYCR, another common motif for OSCs, possibly functions in determining product specificities for the OSCs (Kushiro et al., 2000). In addition, conserved QW motifs of OSCs located at the surface of the enzyme have been proposed to function in stabilizing the enzyme during highly exothermic cyclization reaction (Wendt et al., 1997).

\section{Conclusion and Future Prospects}

Triterpenes are among the largest and diverse classes of phytochemicals with both primary and secondary functions in plants. The cyclization of 2,3oxidosqualene into diverse triterpene scaffolds, catalyzed by OSC is the initial diversifying step in triterpene biosynthesis. Further diversification of triterpene scaffolds is carried out by the tailoring enzymes such as CYP450s, ATs and GTs. With the increasing availability of genomic and transcriptomic sequences, a growing number of plant OSCs are being identified. To date, nearly 90 plant OSCs are biochemically characterized for their product specificities. More than two third of the characterized OSCs are mono-functional and have product specificity towards single triterpene. Rest of the characterized OSCs are multifunctional with triterpene products ranging from 2 to 23 in number. Therefore, the product diversity of the mono-functional OSCs as well as evolution of multifunctional OSCs contributed to the biosynthesis of structurally diverse triterpenes in plants. OSC family has possibly evolved in plant following duplication and neofunctionalization of CAS that plays primary function in plants by supplying biosynthetic precursor cycloartenol for membrane sterols and phytohormones (BRs). To date, most of the characterized OSCs are from angiosperms. Future studies on OSCs from basal plants will clarify this orthologous relationship.

Biosynthesis of triterpenes in plants usually takes place in a particular tissue/organ/developmental stage. Besides, triterpene biosynthesis is induced when plants are subjected to biotic/abiotic stress conditions or treated with elicitors such as MeJA. Similarly, OSC expression in plants is also spatio-temporally regulated. Based on the spatio-temporal accumulation patterns, the role of triterpenes in plant physiology/defense against pathogens is expected. However, the biological role of most of the triterpenes is yet to be determined. Phenotypic analysis of the plants that silence or overexpress an OSC will be helpful to understand the biological function of triterpenes.

OSC initiates cyclization of 2,3-oxidosqualene through protonation of epoxide group, then guides the highly reactive carbocation for a series of cyclization and rearrangement steps, followed by termination of the reaction either through deprotonation or addition of water. Although, crystal structure of plant OSC is yet to be resolved, crystal structure of human LAS and another relevant enzyme from bacterial source (SHC) have provided crucial structural and biochemical insights for the OSC-mediated cyclization reaction. Moreover, directed evolution and sitedirected mutational studies on few OSCs revealed critical amino acids that determine the enzyme function and product specificity. Elucidation of crystal structures of plant OSCs and extending mutagenesis studies on different OSCs will be useful to understand the underlying structural and biochemical basis for diverse product specificities for OSCs. This knowledge is essential for ongoing efforts towards enzyme engineering for conversion of a multifunctional OSC to an accurate mono-functional OSC with desired product specificity and also for developing OSCs with novel product specificities. Since OSCs share high degree of sequence similarity, 
identification of amino acids that determine product specificity for the OSCs will also allow a more accurate prediction of the products for the uncharacterized OSCs.

\section{Acknowledgements}

I thank the Director, Council of Scientific and Industrial Research-Central Institute of Medicinal and Aromatic Plants (CSIR-CIMAP) for providing research facilities. I gratefully acknowledge CSIR and

\section{References}

Abe I (2007) Enzymatic synthesis of cyclic triterpenes Nat Prod Rep 24 1311-31

Amoa Onguéné P, Ntie-Kang F, Lifongo L L, Ndom J C, Sippl W and Mbaze L M (2013) The potential of antimalarial compounds derived from African medicinal plants, part I: a pharmacological evaluation of alkaloids and terpenoids Malar J 12449

Barrero A F, Manzaneda R E A and Manzaneda R R A (1990) Achilleol B: a new tricyclic triterpene skeleton from Achillea odorata L Tetrahedron 46 8161-8168

Biazzi E, Carelli M, Tava A, Abbruscato P, Losini I, Avato P, Scotti C and Calderini O (2015) CYP72A67 catalyzes a key oxidative step in medicago truncatula hemolytic saponin biosynthesis Mol Plant 8 1493-1506

Bishop G J and Koncz C (2002) Brassinosteroids and plant steroid hormone signaling Plant Cell 14 S97-S110

Bode H B, Zeggel B, Silakowski B, Wenzel S C, Reichenbach H and Müller R (2003) Steroid biosynthesis in prokaryotes: identification of myxobacterial steroids and cloning of the first bacterial2,3(S)-oxidosqualene cyclase from the myxobacterium Stigmatella aurantiaca Mol Microbiol 47 $471-481$

Canter P H, Thomas H and Ernst E (2005) Bringing medicinal plants into cultivation: opportunities and challenges for biotechnology Trends Biotechnol 23 180-185

Carelli M, Biazzi E, Panara F, Tava A, Scaramelli L, Porceddu A, Graham N, Odoardi M, Piano E, Arcioni S, May S, Scotti $\mathrm{C}$ and Calderini O (2011) Medicago truncatula CYP716A12 is a multifunctional oxidase involved in the biosynthesis ofhemolytic saponins Plant Cell 23 3070-3081

Chang C H, Wen H Y, Shie W S, Lu C T, Li M E, Liu Y T, Li W H and Wu T K (2013) Protein engineering of oxidosqualenelanosterol cyclase into triterpene monocyclase $\mathrm{Org}$ Biomol Chem 11 4214- 4219
Department of Biotechnology for funding research on triterpene natural products in our laboratory under the Network/Young Scientist Project (BSC0107, BSC0203 and P81 EMR) and IYBA Project (BT/08/ IYBA/2014-13), respectively. I am also thankful to the research scholars and our collaborators for their contributions in triterpene research. I apologize to all the authors, whose work I could not include because of space constraint. Institutional communication number for this article is CIMAP/PUB/2016/26.

Cheng J J, Zhang L J, Cheng H L, Chiou C T, Lee I J and Kuo Y H (2010) Cytotoxic Hexacyclic Triterpene Acids from Euscaphis japonica J Nat Prod 73 1655-1658

Corey E J, Matsuda S P and Bartel B (1993) Isolation of an Arabidopsis thaliana gene encoding cycloartenol synthase by functional expression in a yeast mutant lacking lanosterol synthase by the use of a chromatographic screen Proc Natl Acad Sci U S A 90 11628-11632

Da Silva Ferreira D, Esperandim V R, Toldo M P, Kuehn C C, do Prado Júnior J C, Cunha W R, e Silva M L and de Albuquerque S (2013) In vivo activity of ursolic and oleanolic acids during the acute phase of Trypanosoma cruzi infection Exp Parasitol 134 455-459

Dang T and Prestwich GD (2000) Site-directed mutagenesis of squalene-hopene cyclase: altered substrate specificity and product distribution Chem Biol 7 643-649

De Luca V, Salim V, Atsumi S M and Yu F (2012) Mining the biodiversity of plants: a revolution in the making Science 336 1658-1661

Delis C, Krokida A, Georgiou S, Pena-Rodriguez L M, Kavroulakis N, Ioannou E, Roussis V, Osbourn A E and Papadopoulou K K (2011) Role of lupeol synthase in Lotus japonicus nodule formation New Phytol 189 335346

Domingo V, Lorenzo L, Quilez del Moral J F and Barrero A F (2013) First synthesis of (+)-myrrhanol C, an anti-prostate cancer lead Org Biomol Chem 11 559-562

Dong S H, Zhang C R, Dong L, Wu Y and Yue J M (2011) Onoceranoid-type triterpenoids from Lansium domesticum J Nat Prod 74 1042-1048

Field B and Osbourn AE (2008) Metabolic diversificationindependent assembly of operon-like gene clusters in different plants Science 320 543-547

Field B, Fiston-Lavier A S, Kemen A, Geisler K, Quesneville H and Osbourn A E (2011) Formation of plant metabolic 
gene clusters within dynamic chromosomal regions Proc Natl Acad Sci USA 108 16116-16121

Fukuda N, Shan S, Tanaka H and Shoyama Y (2006) New staining methodology: Eastern blotting for glycosides in the field of Kampo medi-cines J Nat Med $\mathbf{6 0} 21-27$

Garg A, Agrawal L, Misra R C, Sharma S, Ghosh S (2015) Andrographis paniculata transcriptome provides molecular insights into tissue-specific accumulation of medicinal diterpenes BMC Genomics 16659

Gas-Pascual E, Berna A, Bach T J and Schaller H (2014) Plant oxidosqualene metabolism: cycloartenol synthasedependent sterol biosynthesis in Nicotiana benthamiana PLOS ONE 9 e 109156

Gershenzon J and Dudareva N (2007) The function of terpene natural products in the natural world Nat Chem Biol 3 408-414

Ghosh T, Maity T K and Singh J (2011) Antihyperglycemic activity of bacosine, a triterpene from Bacopa monnieri, in alloxan-induced diabetic rats Planta Med 77 804-808

Go Y S, Lee S B, Kim H J, Kim J, Park H Y, Kim J K, Shibata K, Yokota T, Ohyama K, Muranaka T, Arseniyadis S and Suh M C (2012) Identification of marneral synthase, which is critical for growth and development in Arabidopsis Plant J 72 791-804

González-Coloma A, Lopez-Balboa C, Santana O, Reina M and Fraga B M (2011) Triterpene-based plant defences Phytochem Rev 10 245-260

Hart E A, Hua L, Darr L B, Wilson W K, Pang J and Matsuda S P T (1999) Directed evolution to investigate steric control of enzymatic oxidosqualene cyclization. An isoleucine-tovaline mutation in cycloartenol synthase allows lanosterol and parkeol biosynthesis J Am Chem Soc 121 9887-9888

Haubrick L L and Assmann S M (2006) Brassinosteroids and plant function: some clues, more puzzles Plant Cell Environ 29 446-457

Herrera J B R, Wilson W K and Matsuda S P T (2000) A tyrosine-to-threonine mutation converts cycloartenol synthase to an oxidosqualene cyclase that forms lanosterol as its major product J Am Chem Soc 122 6765-6766

Hill R A and Connolly J D (2012) Triterpenoids Nat Prod Rep 29 780-818

Hill R A and Connolly J D (2013) Triterpenoids Nat Prod Rep 30 1028-1065

Jiang Z, Kempinski C, Bush C J, Nybo S E and Chappell J (2016) Engineering triterpene and methylated triterpene production in plants provides biochemical and physiological insights into terpene metabolism Plant

\section{Physiol 170 702-716}

Joubert B M, Hua L and Matsuda S P T (2000) Steric Bulk at Position 454 in Saccharomyces cerevisiae lanosterol synthase influences B-ring formation but not deprotonation Org Lett 2 339-341

Kashyap D, Tuli H S and Sharma A K (2016) Ursolic acid (UA): A metabolite with promising therapeutic potential Life Sci 146 201-213

Kemen A C, Honkanen S, Melton R E, Findlay K C, Mugford S T, Hayashi K, Haralampidis K, Rosser S J and Osbourn A (2014) Investigation of triterpene synthesis and regulation in oats reveals a role for $\beta$-amyrin in determining root epidermal cell patterning Proc Natl Acad Sci U S A 111 8679-8684

Koksal M, Hu H, Coates R M, Peters R J and Christianson D W (2011) Structure and mechanism of the diterpene cyclase ent-copalyl diphosphate synthase Nat Chem Biol 7 431433

Kolesnikova M D, Xiong Q, Lodeiro S, Hua L and Matsuda S P T (2006) Lanosterol biosynthesis in plants Arch Biochem Biophys 447 87-95

Kong L, Li S, Liao Q, Zhang Y, Sun R, Zhu X, Zhang Q, Wang J, Wu X, Fang X and Zhu Y (2013) Oleanolic acid and ursolic acid: novel hepatitis $\mathrm{C}$ virus antivirals that inhibit NS5B activity Antiviral Res 98 44-53

Kurek A, Nadkowska P, Pliszka S and Wolska KI (2012) Modulation of antibiotic resistance in bacterial pathogens by oleanolic acid and ursolic acid Phytomedicine 19 515519

Kushiro T, Shibuya M and Ebizuka Y (1999) Chimeric triterpene synthase. a possible model for multifunctional triterpene synthase J Am Chem Soc 121 1208-1216

Kushiro T, Shibuya M, Masuda K and Ebizuka Y (2000) Mutational studies on triterpene synthases: engineering lupeol synthase into $\beta$-amyrin synthase $J$ Am Chem Soc 122 6816-6824

Lange B M and Ahkami A (2013) Metabolic engineering of plant monoterpenes, sesquiterpenes and diterpenes-current status and future opportunities Plant Biotechnol J 11 169196

Laszczyk M N (2009) Pentacyclic triterpenes of the lupane, oleanane and ursane group as tools in cancer therapy Planta Med 75 1549-1560

Lee K T, Koo S J, Jung S H, Choi J, Jung H J and Park H J (2002) Structure of three new terpenoids, spiciformisins $a$ and $b$, and monocyclosqualene, isolated from the herbs of Ligularia fischeri var. spiciformis and cytotoxicity Arch Pharm Res 25 820-823 
Li Y C, Tian K, Sun L J, Long H, Li L J and Wu Z Z (2016) A new hexacyclic triterpene acid from the roots of Euscaphis japonica and its inhibitory activity on triglyceride accumulation Fitoterapia 109 261-265

Liang C Q, Shi Y M, Li X Y, Luo R H, Li Y, Zheng Y T, Zhang H B, Xiao W L and Sun H D (2013) Kadcotriones AC: tricyclic triterpenoids from Kadsura coccinea $J$ Nat Prod 76 2350-2354

Lin H C, Ding H Y and Wu Y C (1998) Two novel compounds from Paeonia suffructicosa J Nat Prod 61 343-346

Liu Y, Luo S H, Schmidt A, Gershenzon J, Wang G, Sun G L, Grant M, Kuang C, Yang M J, Jing S X, Li C H, Schneider B and Li S H (2016) A geranylfarnesyl diphosphate synthase provides the precursor for sesterterpenoid (C25) formation in the glandular trichomes of Leucosceptrum canum Plant Cell tpc.15.00715

Lodeiro S, Schulz-Gasch T and Matsuda S P T (2005) Enzyme redesign: two mutations cooperate to convert cycloartenol synthase into an accurate lanosterol synthase $\mathrm{J} \mathrm{Am} \mathrm{Chem}$ Soc 127 14132-14133

Lodeiro S, Segura M J R, Stahl M, Schulz-Gasch T and Matsuda S P T (2004) Oxidosqualene cyclase second-sphere residues profoundly influence the product profile ChemBioChem 5 1581-1585

Lodeiro S, Xiong Q, Wilson W K, Kolesnikova M D, Onak C S and Matsuda S PT (2007) An oxidosqualene cyclase makes numerous products by diverse mechanisms: a challenge to prevailing concepts of triterpene biosynthesis J Am Chem Soc 129 11213-11222

Mallavadhani U V, Chandrashekhar M, Nayak V L and Ramakrishna S (2015) Synthesis and anticancer activity of novel fused pyrimidine hybrids of myrrhanone $\mathrm{C}$, a bicyclictriterpene of Commiphora mukul gum resin $\mathrm{Mol}$ Divers 19 745-757

Martin D E, Salzwedel K and Allaway G P (2008) Bevirimat: a novel maturation inhibitor for the treatment of HIV-1 infection Antivir Chem Chemother 19 107-113

Meng F Y, Sun J X, Li X, Yu H Y, Li S M and Ruan H L (2011) Schiglautone A, a new tricyclic triterpenoid with a unique 6/7/9-fused skeleton from the stems of Schisandra glaucescens Org Lett 13 1502-1505

Ming L J and Yin A C (2013) Therapeutic effects of glycyrrhizic acid Nat Prod Commun 8 415-418

Misra R C, Maiti P, Chanotiya C S, Shanker K and Ghosh S (2014) Methyl jasmonate-elicited transcriptional responses and pentacyclic triterpene biosynthesis in sweet basil Plant Physiol 164 1028-1044

Misra R C, Garg A, Roy S, Chanotiya C S, Vasudev P G, Ghosh
S (2015) Involvement of an ent-copalyl diphosphate synthase in tissue-specific accumulation of specialized diterpenes in Andrographis paniculata Plant Sci 240 5064

Miura T, Takagi S and Ishida T (2012) Management of diabetes and its complications with banaba (Lagerstroemia speciosa L.) and corosolic acid Evid Based Complement Alternat Med 2012871495

Moiteiro C, Marcelo Curto M J, Mohamed N, Bailén M, Martínez-Díaz R and González-Coloma A (2006) Biovalorization of friedelane triterpenes derived from cork processing industry byproducts J Agric Food Chem 54 3566-3571

Morita M, Shibuya M, Kushiro T, Masuda K and Ebizuka Y (2000) Molecular cloning and functional expression of triterpene synthases from pea (Pisum sativum) new alphaamyrin-producing enzyme is a multifunctional triterpene synthase Eur J Biochem 267 3453-3460

Moses T, Pollier J, Faizal A, Apers S, Pieters L, Thevelein J M, Geelen D and Goossens A (2015a) Unraveling the triterpenoid saponin biosynthesis of the African shrub Maesa lanceolata Mol Plant 8 122-135

Moses T, Pollier J, Shen Q, Soetaert S, Reed J, Erffelinck M L, Van Nieuwerburgh F C, Vanden Bossche R, Osbourn A, Thevelein J M, Deforce D, Tang K and Goossens A (2015b) OSC2 and CYP716A14v2 catalyze the biosynthesis of triterpenoids for the cuticle of aerial organs of Artemisia annua Plant Cell 27 286-301

Moses T, Pollier J, Thevelein J M and Goossens A (2013) Bioengineering of plant (tri)terpenoids: from metabolic engineering of plants to synthetic biology in vivo and in vitro New Phytol 200 27-43

Mugford S T, Louveau T, Melton R, Qi X, Bakht S, Hill L, Tsurushima T, Honkanen S, Rosser S J, Lomonossoff G P and Osbourn A (2013) Modularity of plant metabolic gene clusters: A trio of linked genes that are collectively required for acylation of triterpenes in oat Plant Cell 25 1078-1092

Mugford S T, Qi X, Bakht S, Hill L, Wegel E, Hughes R K, Papadopoulou K, Melton R, Philo M, Sainsbury F, Lomonossoff G P, Roy A D, Goss R J and Osbourn A (2009) A serine carboxypeptidase-like acyltransferase is required for synthe-sis of antimicrobial compounds and disease resistance in oats Plant Cell 21 2473-2484

Murata J, Roepke J, Gordon H and De Luca V (2008) The leaf epidermome of Catharanthus roseus reveals its biochemical specialization Plant Cell 20 524-542

Mylona P, Owatworakit A, Papadopoulou K, Jenner H, Qin B, Findlay K, Hill L, Qi X, Bakht S, Melton R and Osbourn 
A (2008) Sad3 and Sad4 are required for sapo-nin biosynthesis and root develop-ment in oat Plant Cell 20 201-212

Nützmann H W and Osbourn A (2014) Gene clustering in plant specialized metabolism Curr Opin Biotechnol 26 91-99

Ohyama K, Suzuki M, Kikuchi J, Saito K and Muranaka T (2009) Dual biosynthetic pathways to phytosterol via cycloartenol and lanosterol in Arabidopsis Proc Natl Acad Sci U S A 106 725-730

Orhan I, Sener B, Hashimoto T, Asakawa Y, Ozgüven M and Ayanoðlu F (2002) Iristectorone K, a novel monocyclic triterpene ester from Iris germanica rhizomes growing in Turkey Fitoterapia 73 316-319

Osbourn A, Goss R J and Field R A (2011) The saponins: polar isoprenoids with important and diverse biological activities Nat Prod Rep 28 1261-126

Paddon CJ, Westfall PJ, Pitera DJ, Benjamin K, Fisher K, McPhee D, Leavell MD, Tai A, Main A, Eng D, et al (2013) Highlevel semisynthetic production of the potent antimalarial artemisinin Nature 496 528-532

Papadopoulou K, Melton R E, Leggett M, Daniels M J and Osbourn A E (1999) Compromised disease resistance in saponin-deficient plants Proc Natl Acad Sci U S A 96 12923-12928

Phillips D R, Rasbery J M, Bartel B and Matsuda S P (2006) Biosynthetic diversity in plant triterpene cyclization $\mathrm{Curr}$ Opin Plant Biol 9 305-314

Pichersky E and Gershenzon J (2002) The formation and function of plant volatiles: perfumes for pollinator attraction and defense Curr Opin Plant Biol 5 237-243

Pollier J and Goossens A (2012) Oleanolic acid Phytochemistry 77 10-15

Potze L, Mullauer F B, Colak S, Kessler J H and Medema J P (2014). Betulinic acid-induced mitochondria-dependent cell death is counterbalanced by an autophagic salvage response Cell Death Dis 5 e1169

Pu J X, Xiao W L, Lu Y, Li R T, Li H M, Zhang L, Huang S X, Li X, Zhao Q S, Zheng Q T and Sun H D (2005) Kadlongilactones A and B, two novel triterpene dilactones from Kadsura longipedunculata possessing a unique skeleton Org Lett 7 5079-5082

Qi X, Bakht S, Leggett M, Maxwell C, Melton R and Osbourn A (2004) A gene cluster for secondary metabolism in oat: Implications for the evolution of metabolic diversity in plants Proc Natl Acad Sci USA 101 8233-8238

Reinert D J, Balliano G and Schulz G E (2004) Conversion of squalene to the pentacarbocyclic hopene Chem Biol 11
$121-126$

Retey R (1990) Enzymic reaction selectivity by negative catalysis or how do enzymes deal with highly reactive intermediates? Angew Chem Int Ed 29 355-361

Roy A, and Saraf S (2006) Limonoids: overview of significant bioactive triterpenes distributed in plants kingdom Biol Pharm Bull 29 191-201

Sawai S and Saito K (2011) Triterpenoid biosynthesis and engineering in plants Front Plant Sci 225

Segura M J R, Jackson B E and Matsuda S P T (2003) Mutagenesis approaches to deduce structure-function relationships in terpene synthases Nat Prod Rep 20 304-317

Segura M J, Meyer M M and Matsuda S P T (2000) Arabidopsis thaliana LUP1 converts oxidosqualene to multiple triterpene alcohols and a triterpene diol Org Lett 2 22572259

Shan S, Tanaka H, and Shoyama Y (2001) Enzyme-linked immunosorb-ent assay for glycyrrhizin using antiglycyrrhizin monoclonal antibody and an eastern blotting technique for glu-curonides of glycyrrhetic acid Anal Chem 73 5784-5790

Shang Y, Ma Y, Zhou Y, Zhang H, Duan L, Chen H, Zeng J, Zhou Q, Wang S, Gu W, Liu M, Ren J, Gu X, Zhang S, Wang Y, Yasukawa K, Bouwmeester H J, Qi X, Zhang Z, Lucas W J and Huang S (2014) Biosynthesis, regulation, and domestication of bitterness in cucumber Science 346 10841088

Sharma K and Zafar R (2015) Occurrence of taraxerol and taraxasterol in medicinal plants Pharmacogn Rev 9 19-23

Sheng H and Sun H (2011) Synthesis, biology and clinical significance of pentacyclic triterpenes: a multi-target approach to prevention and treatment of metabolic and vascular diseases Nat Prod Rep 28 543-593

Shi H, Li Z Y and Guo Y W (2005) A new serratane-type triterpene from Lycopodium phlegmaria Nat Prod Res 19 $777-781$

Sivakumar G, Vail D R, Nair V, Medina-Bolivar F and Lay J O Jr (2009) Plant-based corosolic acid: future anti-diabetic drug? Biotechnol J 4 1704-1711

Stohs S J, Miller H and Kaats R (2012) A review of the efficacy and safety of banaba (Lagerstroemia speciosa L.) and corosolic acid Phytother Res 26 317-324

Suzuki H, Achnine L, Xu R, Matsuda S P T and Dixon R A (2002) A genomics approach to the early stages of triterpene saponin biosynthesis in Medicago truncatula Plant J 32 1033-1048 
Suzuki M, Xiang T, Ohyama K, Seki H, Saito K, Muranaka T, Hayashi H, Katsube Y J, Kushiro T, Shibuya M and Ebizuka Y (2006) Lanosterol synthase in dicotyledonous plants Plant Cell Physiol 47 565-571

Tava A, Scotti C and Avato P (2011) Biosynthesis of saponins in the genus Medicago Phytochem Rev 10 459-469

Thimmappa R, Geisler K, Louveau T, O’Maille P and Osbourn A (2014) Triterpene biosynthesis in plants Annu Rev Plant Biol 65 225-257

Thoma R, Schulz-Gasch T, D’Arcy B, Benz J, Aebi J, Dehmlow H, Hennig M, Stihle M and Ruf A (2004) Insight into steroid scaffold formation from the structure of human oxidosqualene cyclase Nature 432 118-122

Wang L Y, Wang N L, Yao X S, Miyata S and Kitanaka S (2003) Euphane and tirucallane triterpenes from the roots of Euphorbia kansui and their in vitro effects on the cell division of Xenopus J Nat Prod 66 630-633

Wendt K U, Lenhart A and Schulz G E (1999) The structure of the membrane protein squalene-hopene cyclase at 2.0 ÚA resolution J Mol Biol 286 175-187

Wendt K U, Poralla K and Schulz G E (1997) Structure and function of a squalene cyclase Science 277 1811-1815

Williams M and Ellis J (2013) Medicinal plants: past, present, and future Plant Cell 251 tpc.113.tt0113
Wink M (2010) Introduction. In: Functions and Biotechnology of Plant Secondary Metabolites (Ed: Wink M) pp 1-20, Wiley-Blackwell

Wozniak L, Skapska S and Marszalek K (2015) Ursolic acid-a pentacyclic triterpenoid with a wide spectrum of pharmacological activities Molecules 20 20614-20641

Xu R, Fazio G C and Matsuda S P (2004) On the origins of triterpenoid skeletal diversity Phytochemistry 65 261-291

Yeats T H and Rose J K (2013) The formation and function of plant cuticles Plant Physiol 163 5-20

Yu F, Thamm A M, Reed D, Villa-Ruano N, Quesada A L, Gloria E L, Covello P and De Luca V (2013) Functional characterization of amyrin synthase involved in ursolic acid biosynthesis in Catharanthus roseus leaf epidermis Phytochemistry 91 122-127

Zhang X, Xiong H and Liu L (2012) Effects of taraxasterol on inflammatory responses in lipopolysaccharide-induced RAW 264.7 macrophages J Ethnopharmacol 141 206-211

Zhao M, Gödecke T, Gunn J, Duan J A and Che C T (2013) Protostane and fusidane triterpenes: a mini-review Molecules 18 4054-4080

Zhou H, Li Y S, Tong X T, Liu H Q, Jiang S H and Zhu D Y (2004) Serratane-type triterpenoids from Huperzia serrata Nat Prod Res 18 453-459. 